\title{
Dielectric Properties of Thin Tantalum Oxide Layers at Solid Tantalum Capacitors
}

\author{
I. Abuetwirat, K. Liedermann \\ Department of Physics \\ Brno University of Technology \\ Czech Republic
}

\begin{abstract}
In this paper MIS (Metal-Insulator-Semiconductor) $\mathrm{Ta}_{2} \mathrm{O}_{5}$ capacitor has been studied in terms of dielectric relaxation with a low frequency dielectric spectroscopy. The results acquired for $\mathrm{Ta}_{2} \mathrm{O}_{5}$ show a relaxation peak in the temperature and frequency range available, $187 \mathrm{~K}-385 \mathrm{~K}, 1 \mathrm{~Hz}-10 \mathrm{MHz}$. The loss peak frequency follows the Arrhenius law dependence with the activation energy of $0.048 \mathrm{eV}$. In conductivity spectra, $\mathrm{Ta}_{2} \mathrm{O}_{5}$ film exhibits a steady-state value at low frequencies and a monotonous increase at high frequencies depending on temperature. The observed conductivity followed a slightly superlinear power law.
\end{abstract}

Keywords-thin oxide film; dielectric properties; HavriliakNegami $(H N)$ equation; electrical conductivity

\section{INTRODUCTION}

Solid tantalum capacitors are increasingly being used in electronic circuitry because of their high volumetric efficiency, reliability, and temperature stability over the range $-55^{\circ} \mathrm{C}$ to $+125{ }^{\circ} \mathrm{C}$ [1]. The construction of tantalum capacitor begins with a fine tantalum powder from pure elemental tantalum metal. The anode of the capacitor is formed from tantalum powder by pressing it into a pellet around a tantalum wire. This is anodized at $\sim 85^{\circ} \mathrm{C}$ to produce a thin dielectric film of amorphous tantalum pent-oxide $\left(\mathrm{Ta}_{2} \mathrm{O}_{5}\right)$. For a $1 \mu \mathrm{F}$ capacitor (manufactured by AVX) with a rated voltage of $50 \mathrm{Vdc}$ the dielectric thickness is $\sim 289 \mathrm{~nm}$. Between the tantalum anode and the $\mathrm{Ta}_{2} \mathrm{O}_{5}$ dielectric a very thin semiconductor layer of tantalum monoxide (TO) forms and this gives the capacitor its polar characteristics [1]. The second electrode is cathode material. The pellet is dipped into an aqueous solution of $\mathrm{Mn}\left(\mathrm{NO}_{3}\right)_{2}$. The pellet is heated till $250{ }^{\circ} \mathrm{C}$ to dry the solution and convert it to manganese dioxide $\left(\mathrm{MnO}_{2}\right)$. The silver paint is applied to the top of the $\mathrm{MnO}_{2}$ to allow a low resistance external connection to the capacitor. Before the application of silver, a coating of graphite solution is applied to eliminate interfacial resistances due to the contact and silver oxide formation at interfacial region [2]. The last step is to enclose the capacitor in epoxy resin.

For measurement of the dielectric properties of capacitors dielectric spectroscopy were used. Alpha-A analyzer with the Quatro Cryosystem were used. Quatro Cryosystem is designed for easy, safe and fully automatic operation enabling computer controlled experiments over several days without supervision as required for low frequency measurements with high resolution and accuracy. These features make them ideal for testing low frequency component such as solid tantalum capacitor.
The dielectric relaxation response was analyzed by the Havriliak-Negami (HN) relaxation function which is a frequency-domain function.

$$
\widehat{\varepsilon}(\omega)=\varepsilon_{\infty}+\frac{\left(\varepsilon_{s}-\varepsilon_{\infty}\right)}{\left[1+\left(j \omega \tau_{0}\right)^{\alpha}\right]^{\beta}}
$$

where $\alpha$ and $\beta$ represent the width (symmetry) and the skewness (asymmetry) of the dielectric loss number $\varepsilon^{\prime \prime}(\omega)$ when viewed in the $\log \left(\varepsilon^{\prime \prime}\right)-\log (\omega)$ plot.

\section{EXPERIMENTAL METHOD}

Figure 1 shows a detail of the measurement setup. For temperature measurement, $1 \mu \mathrm{F}$ solid tantalum capacitor was lined with copper wire to ensure good contact between capacitor and sample cell BDS 1200; the sample cell BDS 1200 was inserted into Cryostat BDS 1100 . Quatro controller BDS 1330 consists of four circuits controlling the sample temperature, the gas temperature, the temperature of the liquid nitrogen in the dewar and the pressure in the dewar. Alpha-A analyzer with a test signal of amplitude $2.2 \mathrm{~V}_{\text {rms }}$ and frequency range from $1 \mathrm{~Hz}$ to $10 \mathrm{MHz}$ was used to measure capacitance and dissipation factor. After gas passes through the gas heater, it flows directly through the vacuum insulated sample by a 2 stage rotary vane vacuum pump, providing thermal isolation by low vacuum $(<10 \mu$ bar $)$ cell mounted in a cryostat.

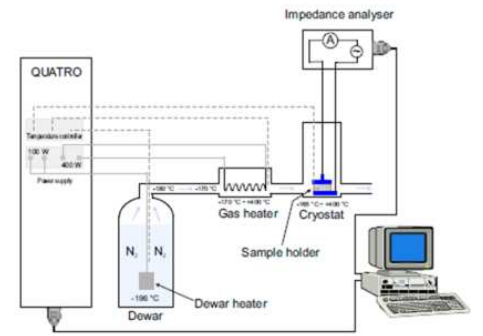

FIGURE I. THE EXPERIMENTAL SETUP [3].

\section{RESULT AND ANALYSIS}

The relative permittivity versus frequency at different temperature range curves for $1 \mu \mathrm{F} / 50 \mathrm{~V}_{\mathrm{dc}}$ tantalum capacitor are shown in Figure. 2. At low frequency, relative permittivity increases slightly with increasing temperature. This suggests that the low frequency behaviour could be attributed to the accumulation of charges at electrodes due to an electrode polarization. The relative permittivity is 27 at $120 \mathrm{~Hz}$ and 304 
K. At higher frequency above $1 \mathrm{MHz}$ and specified temperature $187 \mathrm{~K}$, parasitic effects start to dominate.

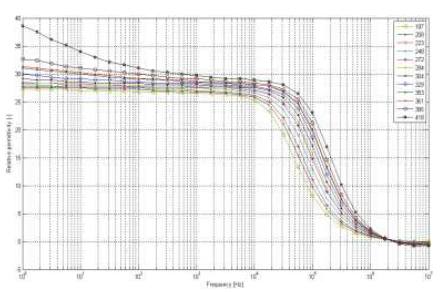

FIGURE II. RELATIVE PERMITTIVITY VS FREQUENCY AS A FUNCTION OF TEMPERATURE.

The relaxation peak starts to appear at frequency about 10 $\mathrm{kHz}$ as shown below. The relaxation peak moves slightly towards the higher frequency with the increasing temperature. The relaxation peak shows almost no amplitude increase with temperature. This behaviour has already been reported for Ta2O5 due to the stability of tantalum raw material and its oxide [4].

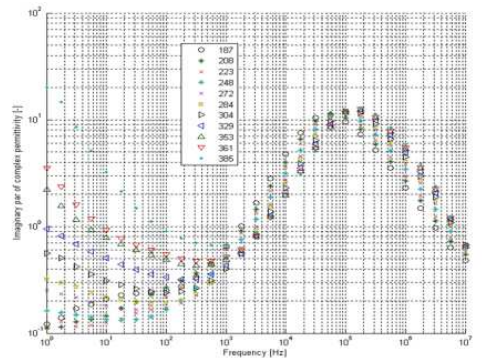

FIGURE III. RELAXATION PEAK VS FREQUENCY AS A FUNCTION OF TEMPERATURE.

In order to evaluate the change in the dielectric loss (dissipation factor) curve we obtained values $\Delta \varepsilon, \alpha_{\mathrm{HN}}, \beta_{\mathrm{HN}}$ and $\tau_{\mathrm{HN}}$ as a function of frequency by fitting the observed values to HN equation.

TABLE I .HAVRILIAK-NEGAMI PARAMETERS OF $1 \mu \mathrm{F} / 50 \mathrm{~V}_{\mathrm{DC}}$ AT DIFFERENT TEMPERATURES: FROM $187 \mathrm{~K}$ TO $418 \mathrm{~K}$.

\begin{tabular}{|c|c|c|c|c|}
\hline $\mathbf{T}[\mathbf{K}]$ & $\boldsymbol{\alpha}_{\mathbf{H N}}[-]$ & $\boldsymbol{\beta}_{\mathrm{HN}}[-]$ & $\Delta \boldsymbol{\varepsilon}[-]$ & $\boldsymbol{\tau}_{\mathbf{0}}[\mathbf{s}]$ \\
\hline 187 & 0.985 & 0.710 & 26.88 & $4.198 \times 10^{-6}$ \\
\hline 208 & 0.980 & 0.724 & 27.14 & $3.370 \times 10^{-6}$ \\
\hline 223 & 0.969 & 0.756 & 27.27 & $2.817 \times 10^{-6}$ \\
\hline 248 & 0.965 & 0.769 & 27.65 & $2.303 \times 10^{-6}$ \\
\hline 272 & 0.971 & 0.766 & 27.98 & $1.965 \times 10^{-6}$ \\
\hline 284 & 0.974 & 0.764 & 28.15 & $1.829 \times 10^{-6}$ \\
\hline 353 & 0.955 & 0.852 & 28.76 & $1.164 \times 10^{-6}$ \\
\hline 361 & 0.950 & 0.873 & 28.84 & $1.105 \times 10^{-6}$ \\
\hline 385 & 0.928 & 0.965 & 28.94 & $9.057 \times 10^{-6}$ \\
\hline 418 & 0.912 & 1 & 29.72 & $7.231 \times 10^{-6}$ \\
\hline
\end{tabular}

Figure 4 show the loss peak frequency follows an Arrhenius law with the activation energy of $0.048 \mathrm{eV}$.

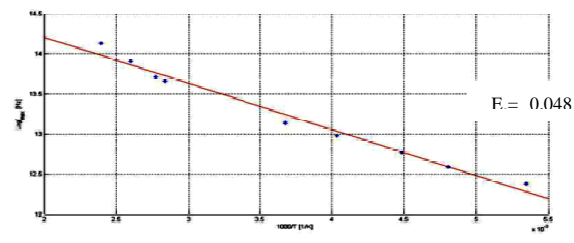

FIGURE IV. ARRHENIUS PLOT OF THE FREQUENCY VALUE OF RELAXATION PEAK.

The capacitance - temperature curves for $1 \mu \mathrm{F} / 50 \mathrm{~V}_{\mathrm{dc}}$ tantalum capacitor are shown in Figure. 5. This figure clearly shows the decrease of the relative permittivity with increased frequency; this is in keeping with specification data of solid tantalum capacitor [1]. This is the main reason for limiting these types of capacitors to low frequency applications.

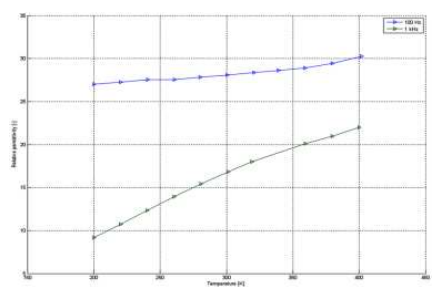

FIGURE V. RELATIVE PERMITTIVITY AS FUNCTION OF TEMPERATURE AND FREQUENCY.

The dissipation factor was $6 \%$ at temperature $300 \mathrm{~K}$ and frequency $100 \mathrm{~Hz}$. At this frequency, losses are at an acceptable level; for the temperature $418 \mathrm{~K}$, i.e., above the normal working temperature, the loss increases considerably. At higher frequency and low temperature dissipation factor increased considerably.

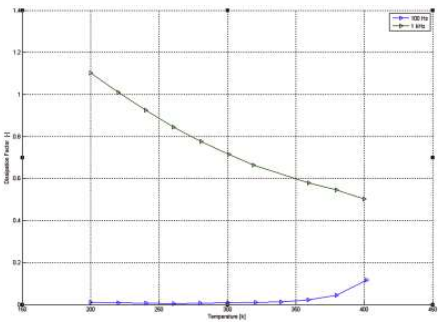

FIGURE VI. DISSIPATION FACTOR AS FUNCTION OF TEMPERATURE AND FREQUENCY.

Figure 7 shows the frequency dependence of electrical conductivity at various temperatures for $1 \mu \mathrm{F} / 50 \mathrm{~V}_{\mathrm{dc}}$ tantalum capacitor. The electrical conductivity depends on frequency according to the "universal dynamic response" [4] and can be related as

$$
\sigma(\omega)=\sigma_{d c}+A . \omega^{n}
$$

where $\mathrm{A}$ is the temperature dependent parameter and the exponent $\mathrm{n}$ is a characteristic parameter representing the many body interactions of the electrons, other charges and impurities. It varies from 0 to 1 . For all temperatures, the conductivity exhibits a steady increase in low frequency regime. Above a characteristic frequency, the conductivity increases with increase in frequency with the characteristic $\omega^{\mathrm{n}}$ dependence. 


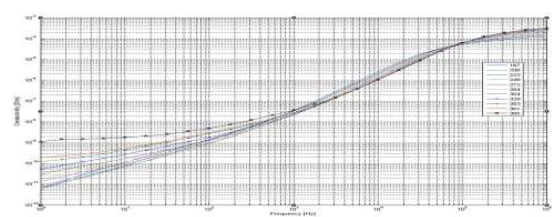

FIGURE VII. THE REAL PART OF THE AC CONDUCTIVITY AS A FUNCTION OF FREQUENCY AT DIFFERENT TEMPERATURES.

\section{CONCLUSIONS}

1. The low frequency behaviour of the relaxation peak could be attributed to an electrode polarization mechanism related to the accumulation of mobile charges at electrodes.

2. The relaxation peak in $\mathrm{Ta}_{2} \mathrm{O}_{5}$ follows the Arrhenius law dependence with the activation energy of $0.048 \mathrm{eV}$.

3. Capacitance and dissipation factor as function of temperature performance curves for tantalum $\left(1 \mu \mathrm{F} / 50 \mathrm{~V}_{\mathrm{dc}}\right)$ capacitor clearly show the capabilities and limitations of tantalum capacitors at low temperature and high frequency.

\section{REFERENCES}

[1] MATHEW, T., Measurement of the low temperature electrical properties of solid tantalum capacitor. Cryogenics 41, PP. 285-288, 2001.

[2] HOLMAN, B. The electrical characterization of tantalum capacitor as MIS devices, [Dissertation thesis], August 2008, Clemson University, South Carolina, USA.

[3] FRUBING, P. Dielectric spectroscopy, 2011, University of Potsdam, Institute of Physics, Advanced Lab, Germany.

[4] SETHI, G., OLSZTA, M., LI, J., SLOPPY, J., HORN, M., DICKEY, E. LANAGAN, M., Structure and dielectric properties of amorphous tantalum pent-oxide thin film capacitors. Annual Report, Conference on Electrical Insulation and Dielectric Phenomena. PP. 815-818, 2007. 